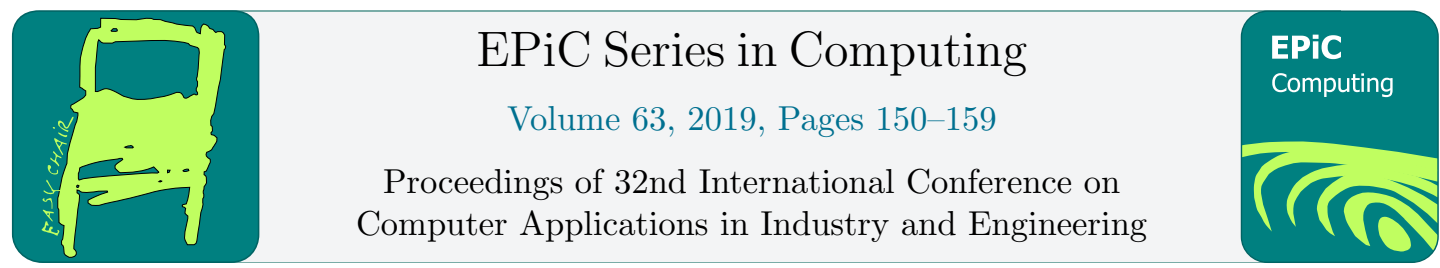

\title{
Descending auction model using mari considering the consumer market
}

\author{
Tomoki Morimoto ${ }^{1}$ and Satoshi Takahashi ${ }^{1}$ \\ The University of Electro-Communications, Chofu, Tokyo, Japan \\ t.1102.book@gmail.com, stakahashi@uec.ac.jp
}

\begin{abstract}
Japanese flower markets employ a descending auction with special rule called "mari". In the descending auction, seller offers an initial price which is enough high. The auction decrease the price when a buyer stops. Then the auction decides the buyer as a winner and allocates the item to the buyer. The mari is a transaction spped up rule, which the buyers without the winner choose to join a coalition for buying the items as the winner's price. Our study extend this model to widely market including consumer transaction. We evaluate our proposed model by simulations. The result shows that our model reduce the number of auctions compered with the previous model.
\end{abstract}

\section{Introduction}

In recent years, with the spread of the Internet, it has become possible to conduct auctions easily, and economic transactions by auctions have become familiar. There are many types of auctions depending on the number of goods sold and purchased, and the method of price determination. For example, in the case of one kind of single goods, there are auctions where the buyer bids many times and raises the price gradually, and conversely, the auction which the price is lowered gradually and the buyer who made the first bid buys. The Japanese flower market uses a special auction method. It uses a slightly modified version of the standard descending auction. The auction is currently conducted using a computer, and a large display board in front of the auction hall displays information such as the number and price of goods exhibited at the auction. When the auction starts, the offer price is gradually lowered, and the buyer presses a button when the price he wants to buy is reached. The first buyer to press a button can buy goods at the stopped price [1]. In the Japanese flower market, there is a rule "mari" which allows other buyers to buy goods at the same price as the first winner if the buyer who won the auction purchases the goods and the goods still remain[2].

When buying goods by mari, the payment is the same price as the first winner of the down auction. Since the buyers participating in mari are the buyers who can not win in the auction, the payment amount is larger than their own bids. When using mari, the buyer's gain may be negative, such as if the bid is higher than her valuation or if the initial winner pays too much. On the other hand, such a market is a part of the economic transaction including procurement

Q. Yuan, Y. Shi, L. Miller, G. Lee, G. Hu and T. Goto (eds.), CAINE 2019 (EPiC Series in Computing, vol. 63), pp. 150-159 
process and customer transaction process, and the profit of the buyer is represented by the sum of the profit from the auction and the profit from consumer market. Therefore, we extend the standard descending auction model with mari to a model that includes procurement process and customer transaction process, and examines the efficiency of trading speed and the allocation efficiency of goods by mari participation.

We describe the composition of this paper. In the second chapter, we explain desending auction and mari. In the third chapter, we extend the model of the existing research and propose the model including the consumer market. In chapter 4 , to analyze the model, we show some numerical simulation result.

\section{Descending auction and "mari"}

An auction is one of the economical environments which decides a price of good and an allocation to buyers. We consider a single item multi unit auction, which a seller wish to sell multi unit of the single object to buyers. An auction mechanism decides both an allocation of items to buyers and a payment of each buyer. Our study focuses on a descending auction mechanism. The procedure of a standard descending auction is as follows.

step1 The seller provides the multi unit single item to the auction with initial price. The item price keeps falling down from the initial price during the auction.

step2 If a first buyer bids an offer to stop the price, the seller fixes the item price and allocates the item to the first buyer. In this time, the first buyer is called the first winner.

In this auction mechanism, only one winner can be decided at each auction, and it is necessary to repeat the auction until there are no items or no buyers. To solve this issue, Japanese flower markets employ a special rule called "mari" where buyers without the winner in the previous auction can apply for purchasing the remain items at the same price as in the previous auction. When there are a lot of number of buyers participating in mari, i.e. the number of remain items is less than the number of mari participators, the mari rule decides the winners at uniform random. The procedure of a standard descending auction with mari [3] is:

step1 The seller provides the multi unit single item to the auction with initial price. The item price keeps falling down from the initial price during the auction.

step2 If a first buyer bids an offer to stop the price, the seller fixes the item price and allocates the item to the first buyer. In this time, the first buyer is called the first winner.

step3 After the decision of first winner, the seller asks to other buyers whether you purchase the item at the same price of first winner or not.

step4 If the number of items grater than or equal to the number of mari participators, all participators get the item with the same price of the first winner. Otherwise the seller select the winner at random.

step5 If goods remain, a standard descending auction is continued.

If the number of mari participators is few, the seller needs to repeat the descending auction until the goods remain. To repeat the descending auction is not efficient in context of time. On the other hand, it is known that mari rule decreasing the allocation efficiency [3]. These two efficiency relationship is a tradeoff. In this study, we focus on an efficiency of trading time. 
When the buyer participates to mari, the buyer consider an own utility. If the buyer purchase the item with the first winner's price, she may get a negative utility in this auction, i.e. the price will exceed her valuation. The rational buyers will participate to mari when her utility will nonnegative. However, in the flower market, the procurement process is only part of economy. We should consider the transaction between the buyer and customers. The buyer's utility is a sum of a utility from the auction and a utility of transaction to customers. Considering the total utility in the economy increases the incentive to participate to mari.

\section{Descending auction model using mari considering the consumer market}

We introduce a procurement economy including the auction and transaction to customers. The auction consists of a seller who wishes to sell $k$ homogeneous goods and $n$ single minded buyers. Let $N=\{1,2, \ldots, n\}$ be a set of buyers. A buyer $i \in N$ has a valuation $v_{i} \in \mathbb{R}_{+}$for a good. Let the buyer $i$ 's bidding strategy be $\beta_{i} \in \mathbb{R}_{+}$which represents a distance between a bid value and the valuation $v_{i}$. For example, if $\beta_{i}$ is greater than 1 , the buyer tells an overbid, and if $\beta_{i}$ less than 1 , the buyer tells an underbid. Thus buyer $i$ 's bid value is $\beta_{i} v_{i}$. Let $r \in \mathbb{R}_{+}$be the reserved price, which is the price that the seller will not sell if it is less than this price. Let $\boldsymbol{x} \in\{0,1\}^{n}$ be an allocation vector, if $x_{i}=1$, the buyer $i$ got a good. Let $p_{i} \in \mathbb{R}_{+}$be a price where the buyer $i$ pays at the auction.Particularly, let $p \in \mathbb{R}_{+}$be a price where the first descending auction winner pays.

We consider a following transaction model between the buyer and customers. The buyer $i$ has a sales price $s_{i} \in \mathbb{R}_{+}$to the customers. Let the buyer $i$ 's sales strategy be $\gamma_{i}\left(\beta_{i}, v_{i}, p_{i}\right) \in \mathbb{R}_{+}$. The sales strategy represents how far from the sales price in which the buyer sells. The final sales price is $\gamma_{i}\left(\beta_{i}, v_{i}, p_{i}\right) s_{i}$. The probability of a sale a good of the buyer $i$ is represented by $q_{i}\left(\beta_{i}, v_{i}, p_{i}, \gamma_{i}, s_{i}\right) \in[0,1]$, and $\lambda_{i} \in[0,1]$. By the definition, buyer $i$ 's sales strategy $\gamma_{i}$ is influenced by own bid and payment at the auction side. Since we are widely considering the sourcing economy, we can discuss strategies to bid higher than the valuation to buy reliable good. It shows that even if a negative utility occurs in an auction, it can be transferred to the sale price of a good. From the definition, $q_{i}$ depends on the selling price and the market value. Since we know the allocation and prices of all goods from the auction results, we can predict the number and price of goods in the consumer market. However, when a buyer think $q_{i}$, the auction is not finished and she does not know the bid of other buyers, so she predicts the market price only by the buyer's own bid and payment.

Descending auction using mari is defined as follows:

Input : For each buyer $i \in N$, number of goods $k$, bidding strategy $\beta_{i}$, valuation $v_{i}$, reserved price $r$, sales price $s_{i}$, and acceptable sales probability $\lambda_{i}$ for the buyer $i$.

Output : A payment vector $\boldsymbol{p}$ and an allocation vector $\boldsymbol{x}$.

step1 In the descending auction, suppose the buyer $c$ with the highest bid $\beta_{c} v_{c}$ is winner. $p \leftarrow \beta_{c} v_{c}$.

step2 (i) If $r>p$,

End of auction.

(ii) Else,

Take $p_{c} \leftarrow p, x_{c} \leftarrow 1, k \leftarrow k-1$ and remove the buyer $c$ from $N$, i.e. $N \leftarrow N \backslash\{c\}$. 
step3 Select a set $M$ of mari paticipants from $N$.

(i) If $|M|>k$, Choose a mari winner subset $M^{\prime} \subseteq M$ with $\left|M^{\prime}\right|=k$.

Take $p_{i}=p, x_{i}=1 \forall i \in M^{\prime}$.

End of auction.

(ii) Else,

Take $p_{i}=1, x_{i}=1 \forall i \in M, k \leftarrow k-|M|$, and $N \leftarrow N \backslash(M \cup\{c\})$.

step4 Repeat the following while $k>0$.

(a) Each bidder $i \in N$ redetermines own bidding strategy $\beta_{i}$.

(b) Buyer $c^{\prime}$ with the highest bid $\beta_{c^{\prime}} v_{c^{\prime}}$ won auction. $p \leftarrow \beta_{c^{\prime}} v_{c^{\prime}}$

(i) If $r>p$,

End of auction.

(ii) Else,

Take $p_{c^{\prime}} \leftarrow p, x_{c^{\prime}} \leftarrow 1, k \leftarrow k-1$ and remove the buyer $c^{\prime}$ from $N$.

At the auction, the buyer determines the following four strategies:

1. bidding strategy $\beta$ in step1,

2. mari participation strategy,

3. bidding strategy $\beta$ in step4,

4. sales strategy $\gamma$ for the consumer market.

An expected payoff $U_{i}$ of the buyer $i$ at the end of the auction can be expressed as follows.

$$
U_{i}=\left(v_{i}-p_{i}\right)+q_{i}\left(\beta_{i}, v_{i}, p_{i}, \gamma_{i}, s_{i}\right)\left(\gamma_{i}\left(\beta_{i}, v_{i}, p_{i}\right) s_{i}-v_{i}\right) .
$$

The buyer's expected payoff is the sum of gains from the auction and the consumer market. In this model, it is considered that the good's valuation remains even though it can not be sold.

We describe how to decide to participate in mari. If the buyer $i$ participates to mari, her payment $p_{i}$ is fixed to $p$. Then the buyer $i$ decides on a sales strategy that maximizes gains as $\operatorname{argmax}\left\{U_{i}\right\}$. $\gamma_{i} \in \mathbb{R}_{+}$

The conditions of mari participation are the follows: (1) $U_{i} \geq 0$ and (2) $q_{i}\left(\beta_{i}, v_{i}, p, \gamma_{i}, s_{i}\right) \geq$ $\lambda_{i}$. The buyer joins mari when the expected payoff becomes nonnegative and the probability of selling the goods at that time is higher than the acceptable sales probability.

\section{Analysis of the model}

\subsection{Definitions for analyzing the model}

Definition 1. A total surplus of the auction is the sum of the buyers' and seller's payoffs, which is

$$
\sum_{i \in N}\left(v_{i}-p_{i}\right) x_{i}+\sum_{i \in N} p_{i} x_{i}
$$


The following two criteria measure a trade speed and allocation efficiency of goods.Kitahara and Ogawa [3] used this to analyze the descending auction model with mari.

Definition 2. Efficiency loss rate (LR) is the ratio of the allocation efficiency of the goods lost by mari and the maximum surplus.

$$
L R=\frac{\text { maximum surplus }- \text { surplus when allocated using mari }}{\text { maximum surplus }}
$$

If $L R=0$, it indicates the most efficient allocation situation when allocated using mari and if $L R=1$, it indicates a situation where all participants did not get anything when allocated using mari.

$L R$ is used to confirm the allocation efficiency of goods. If it is small, it indicates that the auction achieves efficiently allocation. The most efficient allocation is that $k$ goods are allocated to the buyer by descending order of valuation. If thee auction employs mari, the auction may not be able to allocate goods in order from high valued buyers.

Definition 3. Round reduction rate $(R R)$ indicates the ratio between the number of goods allocated by mari and the number of goods remaining at start step3.

$$
R R=\frac{\text { Number of goods sold by mari }}{\text { Number of goods remaining at start step3 }}
$$

If $R R=0$, then there is no mari participant and all goods are sold in standard descending auctions, if $R R=1$, it indicatethe situation where all goods are sold in mari.

$R R$ is used to confirm a trade speed.If it is large,it indicates that trade speed is fast.If there is no mari participant, seller repeats descending auction $k$ times. The number of descending auctions can be reduced by selling goods in mari.

\subsection{Numerical simulation}

We create a simulator for the proposed model and Kitahata and Ogawa model [3]. The difference between the two models is the buyer's strategy. The proposed model calculates $\max _{\gamma} U_{i}$ using successive least squares method when deciding whether to join mari. Kitahara and Ogawa model calculates expected payoff and use them to determine mari participation strategies.

First, $v_{i}$ is uniformly selected from a closed interval $[0,1], s_{i}$ is uniformly selected from a closed interval $\left[v_{i}, 1\right]$, and $\lambda_{i}$ is uniformly selected from a closed interval $[0,1]$. When considering whether to participate in mari, the values on which $q_{i}$ depends are fixed except for $\gamma$. Then we assume the probability of selling goods as follows.

$$
\begin{aligned}
q_{i}= & \left\{\begin{array}{l}
-a \gamma+b \\
0(\text { if }-a \gamma+b<0),
\end{array}\right. \\
& a>0, b \in[0,1], \gamma>0 .
\end{aligned}
$$

The parameter $a$ indicates how much the probability of selling a good changes when the buyer $i$ changes the price of the good, and the parameter $b$ indicates the probability of selling when the good's price is 0 . This is an assumption that simply raising the price reduces the probability of selling a good. We find $\gamma$ analytically. The mari participation condition holds:

$$
\begin{array}{r}
\max _{\gamma}\left\{U_{i}(\gamma)=\left(v_{i}-p_{i}\right)+(-a \gamma+b)\left(\gamma s_{i}-v_{i}\right)\right\} \\
\Leftrightarrow \min _{\gamma}\left\{-U_{i}(\gamma)=-\left(v_{i}-p_{i}\right)-(-a \gamma+b)\left(\gamma s_{i}-v_{i}\right)\right\} .
\end{array}
$$


Transform $-U_{i}(\gamma)=a s_{i} \gamma^{2}-\left(a v_{i}+b s_{i}\right) \gamma+b v_{i}-\left(v_{i}-p_{i}\right)$. Differentiate the objective function and consider the minimum solution.

$$
\left(-U_{i}(\gamma)\right)^{\prime}=2 a s_{i} \gamma-\left(a v_{i}+b s_{i}\right)=0
$$

So the minimum $\gamma$ is

$$
\gamma=\frac{a v_{i}+b s_{i}}{2 a s_{i}}=\frac{b}{2 a}+\frac{v_{i}}{2 s_{i}}
$$

Because of $0 \leq v_{i} \leq 1,0 \leq s_{i} \leq 1$

$$
\frac{b}{2 a} \leq \gamma \leq \frac{b}{2 a}+\frac{1}{2}
$$

Assign $\gamma$ to the objective function.

$$
\begin{aligned}
& a s_{i}\left(\frac{a v_{i}+b s_{i}}{2 a s_{i}}\right)^{2}-\left(a v_{i}+b s_{i}\right)\left(\frac{a v_{i}+b s_{i}}{2 a s_{i}}\right)+b v_{i}-\left(v_{i}-p_{i}\right) \\
= & \frac{\left(a v_{i}+b s_{i}\right)^{2}}{4 a s_{i}}-\frac{\left(a v_{i}+b s_{i}\right)^{2}}{2 a s_{i}}+b v_{i}-\left(v_{i}-p_{i}\right) \\
= & -\frac{\left(a v_{i}-b s_{i}\right)^{2}}{4 a s_{i}}-\left(v_{i}-p_{i}\right) .
\end{aligned}
$$

Therefore, the maximum $U_{i}$ under this assumption is

$$
U_{i}=\left(v_{i}-p_{i}\right)+\frac{\left(a v_{i}-b s_{i}\right)^{2}}{4 a s_{i}}
$$

This indicates that the buyer can lose up to $\frac{\left(a v_{i}-b s_{i}\right)^{2}}{4 a s_{i}}$.

For example, if $a=\frac{1}{2}, b=1$, then $q_{i}=-\frac{1}{2} \gamma+1(\gamma \in[0,2])$. This is the case where the buyer $i$ thinks that if $\gamma=0$ the goods will surely sell and $\gamma=2$ they will never sell. The sales strategy of the buyer $i$ is $1 \leq \gamma \leq 1.5$. At this time, $0.25 \leq q_{i} \leq 0.5$, so it is known that buyers who participated in mari under this assumption have a low probability of selling goods.

We assume four other probabilities of selling goods. First,

$$
q_{i}=\left\{\begin{array}{l}
-\gamma^{2}+2 \gamma \\
0\left(\text { if }-\gamma^{2}+2 \gamma<0\right) .
\end{array}\right.
$$

This is the case where the buyer $i$ thinks that if $\gamma=1$, the goods will surely sell, and if $\gamma=0,2$, it will never sell. If goods are significantly cheap, consumers will not buy with anxiety. This is an assumption that considers not only the price but also the buyer's anxiety. Second,

$$
q_{i}=\left\{\begin{array}{l}
-0.8 \gamma^{2}+1.6 \gamma \\
0\left(\text { if }-0.8 \gamma^{2}+1.6 \gamma<0\right) .
\end{array}\right.
$$

The assumption is that the probability of selling goods is reduced in the same situation as above. Third,

$$
q_{i}=\left\{\begin{array}{l}
\left(\frac{1}{2}\right)^{\gamma} \\
0\left(\operatorname{if}\left(\frac{1}{2}\right)^{\gamma}<0\right)
\end{array}\right.
$$


This is the assumption that if you raise the price, the probability of selling the goods falls gently. Last,

$$
q_{i}=\left\{\begin{array}{l}
\frac{0.8}{1+\mathrm{e}^{50(\gamma-1)}} \\
0\left(\mathrm{if}_{\frac{0.8}{1+\mathrm{e}^{50(\gamma-1)}}}<0\right) .
\end{array}\right.
$$

This is known as a sigmoid function. This is the assumption that the probability of selling goods suddenly drops below a certain price. It is assumed that similar goods are displayed in order of price on the net.

Using these five sales probabilities, we simulate a descending auction with mari. The experimental environment is as follows. Table 1 shows an environment of our simulator coded by Python 3.6.3.

Table 1: The experimental environment

\begin{tabular}{ccc}
\hline CPU & Memory & OS \\
\hline $2.6 \mathrm{HHz}$ Intel Core i5 & 8 GB 1600 MHz DDDR3 & macOS Sierra 10.12.6 \\
\hline
\end{tabular}

First, we prepare 10 instances for the number of goods $k$ and the number of buyers $n$. After that, set the other inputs as shown in Table 2 below. The bidding strategy is the same as the existing research.

Table 2: Input to the experiment

\begin{tabular}{cccc}
\hline & Existing model[3] & Proposed model 1 & Proposed model2 \\
\hline$v_{i}$ & $\mathrm{U}[0,1]$ & $\mathrm{U}[0,1]$ & $\mathrm{U}[0,1]$ \\
$\beta_{i}$ & $\frac{n-k}{n}$ & $\frac{n-k}{n}$ & $\frac{n-k}{n}$ \\
$r$ & 0 & 0 & 0 \\
$q_{i}$ & & $q=-\frac{\gamma}{2}+1$ & $q=-\frac{\gamma}{2}+1$ \\
$s_{i}$ & & $\mathrm{U}\left[v_{i}, 1\right]$ & $\mathrm{U}\left[v_{i}, 1\right]$ \\
$\lambda_{i}$ & & $\mathrm{U}[0,1]$ & $\lambda=0$ \\
\hline
\end{tabular}

\begin{tabular}{cccc}
\hline & Proposed model 3 & Proposed model4 & Proposed model 5 \\
\hline$v_{i}$ & $\mathrm{U}[0,1]$ & $\mathrm{U}[0,1]$ & $\mathrm{U}[0,1]$ \\
$\beta_{i}$ & $\frac{n-k}{n}$ & $\frac{n-k}{n}$ & $\frac{n-k}{n}$ \\
$r$ & 0 & 0 & 0 \\
$q_{i}$ & $q=-\gamma^{2}+2 \gamma$ & $q=-\gamma^{2}+2 \gamma$ & $q=-0.8 \gamma^{2}+1.6 \gamma$ \\
$s_{i}$ & $\mathrm{U}\left[v_{i}, 1\right]$ & $\mathrm{U}\left[v_{i}, 1\right]$ & $\mathrm{U}\left[v_{i}, 1\right]$ \\
$\lambda_{i}$ & $\mathrm{U}[0,1]$ & $\lambda=0$ & $\mathrm{U}[0,1]$ \\
\hline
\end{tabular}

I input the values in Table 2 into the existing research model and the proposed model and compare them. We also compare cases where we do not consider acceptable sales probability among the proposed models. The number of LR, RR, and mari participants (MP for short) are calculated for each model for the number of goods of 10 instances and the number of buyers. That is Tables 3 and 4 . The values in Tables 3 and 4 are each 100 instances created and the average value written with three significant figures. In order to eliminate the bias, valuation $v_{i}$, sales price $s_{i}$, and acceptable sales probability $\lambda_{i}$ are made the same value in the pattern of the number of same goods and the number of buyers. 


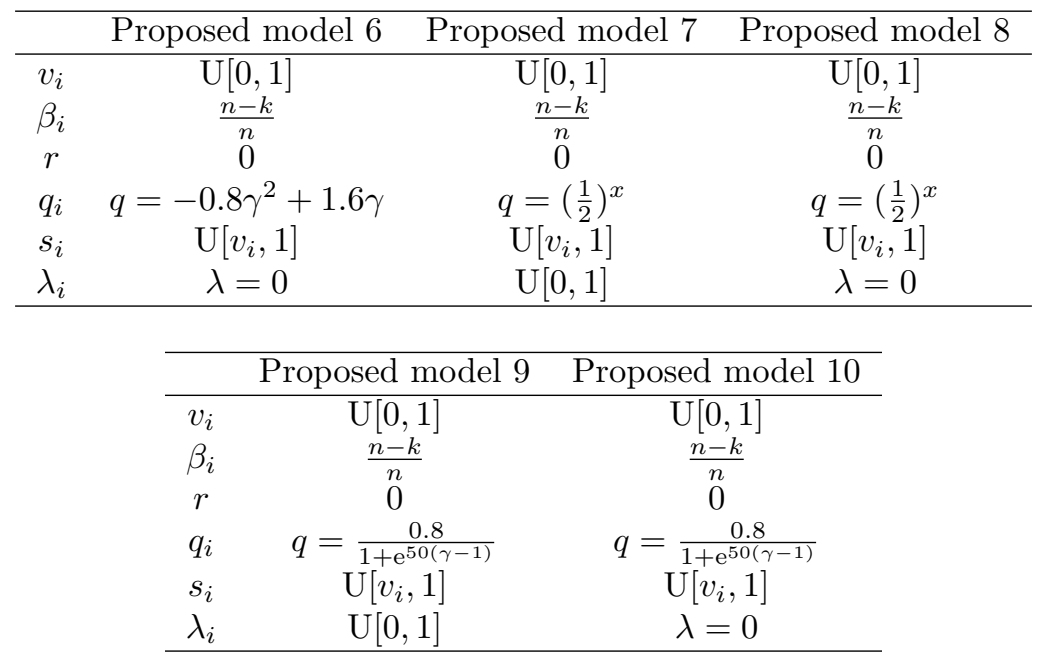

\subsection{Discussion}

From the experiments, our model has faster transaction speed and less allocation efficiency compared with previous model. The reason for the increase in mari participants is considered to be that the conditions for mari participation were relaxed by the incentive to purchase goods at a price that exceeds the valuation by considering the consumer market. It is clearly understand that the decreasing allocation efficiency is caused by the increasing the number of mari participants. If the number of mari participants is greater than the number of goods, the auction decides the winner at random. Thus the probability that a buyer with a high valuation will win is reduced. This result is as same as previous research.

The number of mari participants in proposed model 1 is smaller than in previous research model, and the number of mari participants in proposed model 2 is larger than previous model. Since the difference between the proposed model 1 and 2 is whether or not to consider the sales acceptance probability, the proposed model 2 participates in mari if the expected gain is not negative. Since the data of the instance uses the same value, in the proposed model 1 , it can be seen that there are many buyers whose probability of selling a good has not reached the sales acceptance probability even if the expected gain is not negative. It is as same as proposed model 7 . The proposed models $3,4,5,6,9$, and 10 have more mari participants than existing research models, even considering the sales tolerance. It can be seen that the assumption of $q_{i}$, the probability of selling goods, is greatly influenced.

\section{Conclusion}

In this research, we proposed descending auction model using mari considering the consumer market, and confirm the difference between the existing research model and the proposed model by simulation. As a result, under the assumption, it was found how much the buyer could pay at the auction site than the valuation of the goods. The simulation also showed that almost all goods are sold in mari in the proposed model. we were able to confirm the properties of mari in a model closer to reality than existing research.

As a future works, it is possible to make $q$ different value depending on the buyer and extend it to a model to consider how the buyer participates in the auction. Also, it is considered to 
Table 3: Simulation result

\begin{tabular}{|c|c|c|c|c|c|c|}
\hline & & $\mathrm{k}=2, \mathrm{n}=3$ & $\mathrm{k}=2, \mathrm{n}=4$ & $\mathrm{k}=3, \mathrm{n}=4$ & $\mathrm{k}=2, \mathrm{n}=5$ & $\mathrm{k}=3, \mathrm{n}=5$ \\
\hline \multirow{3}{*}{ Existing model [3] } & LR & 0.0186 & 0.0101 & 0.0139 & 0.00994 & 0.00956 \\
\hline & $\mathrm{RR}$ & 0.700 & 0.670 & 0.765 & 0.760 & 0.730 \\
\hline & MP & 0.98 & 1.03 & 1.74 & 1.29 & 1.76 \\
\hline \multirow{3}{*}{ Prop. model 1} & LR & 0.499 & 0.0373 & 0.0534 & 0.0540 & 0.0830 \\
\hline & $\mathrm{RR}$ & 0.580 & 0.570 & 0.495 & 0.530 & 0.555 \\
\hline & MP & 0.710 & 0.780 & 1.01 & 0.880 & 1.19 \\
\hline \multirow{3}{*}{ Prop. model 2} & LR & 0.0665 & 0.0794 & 0.0905 & 0.0925 & 0.112 \\
\hline & $\mathrm{RR}$ & 0.99 & 0.99 & 0.99 & 0.98 & 0.99 \\
\hline & MP & 1.79 & 2.26 & 2.82 & 2.70 & 3.41 \\
\hline \multirow{3}{*}{ Prop. model 3} & LR & 0.103 & 0.124 & 0.105 & 0.141 & 0.152 \\
\hline & $\mathrm{RR}$ & 0.960 & 0.980 & 0.930 & 1.00 & 0.955 \\
\hline & MP & 1.56 & 2.27 & 2.25 & 2.85 & 2.92 \\
\hline \multirow{3}{*}{ Prop. model 4} & LR & 0.0832 & 0.124 & 0.105 & 0.137 & 0.137 \\
\hline & $\mathrm{RR}$ & 1.00 & 1.00 & 1.00 & 1.00 & 1.00 \\
\hline & MP & 1.91 & 2.82 & 2.94 & 3.56 & 3.77 \\
\hline \multirow{3}{*}{ Prop. model 5} & LR & 0.0487 & 0.107 & 0.0904 & 0.134 & 0.140 \\
\hline & $\mathrm{RR}$ & 0.87 & 0.90 & 0.82 & 0.93 & 0.87 \\
\hline & MP & 1.21 & 1.69 & 1.85 & 2.16 & 2.33 \\
\hline \multirow{3}{*}{ Prop. model 6} & LR & 0.0980 & 0.0994 & 0.0864 & 0.144 & 0.140 \\
\hline & $\mathrm{RR}$ & 1.00 & 1.00 & 1.00 & 1.00 & 1.00 \\
\hline & MP & 1.88 & 2.70 & 2.93 & 3.36 & 3.77 \\
\hline \multirow{3}{*}{ Prop. model 7} & LR & 0.0452 & 0.0400 & 0.0422 & 0.0553 & 0.0613 \\
\hline & $\mathrm{RR}$ & 0.500 & 0.460 & 0.380 & 0.480 & 0.395 \\
\hline & MP & 0.53 & 0.56 & 0.77 & 0.64 & 0.85 \\
\hline \multirow{3}{*}{ Prop. model 8} & LR & 0.0749 & 0.0898 & 0.107 & 0.0967 & 0.115 \\
\hline & $\mathrm{RR}$ & 1.00 & 0.990 & 0.995 & 0.990 & 0.995 \\
\hline & MP & 1.86 & 2.40 & 2.87 & 2.91 & 3.54 \\
\hline \multirow{3}{*}{ Prop. model 9} & LR & 0.0682 & 0.0970 & 0.103 & 0.130 & 0.135 \\
\hline & $\mathrm{RR}$ & 0.920 & 0.920 & 0.865 & 0.890 & 0.870 \\
\hline & MP & 1.39 & 1.72 & 2.01 & 2.08 & 2.39 \\
\hline \multirow{3}{*}{ Prop. model 10} & LR & 0.0813 & 0.111 & 0.105 & 0.0993 & 0.108 \\
\hline & $\mathrm{RR}$ & 1.00 & 1.00 & 0.995 & 1.00 & 0.990 \\
\hline & MP & 1.86 & 2.45 & 2.89 & 2.97 & 3.54 \\
\hline
\end{tabular}

change the bidding strategy $\beta$ not to depend only on the number of goods $k$ and the number of buyers $n$, but to change the bid considering the consumer market.

\section{Acknowledgement}

This research was partially supported by Grants-in-Aid for Scientific Research (C) 26330025. 
Table 4: Simulation result

\begin{tabular}{|c|c|c|c|c|c|c|}
\hline & & $\mathrm{k}=4, \mathrm{n}=5$ & $\mathrm{k}=4, \mathrm{n}=20$ & $\mathrm{k}=10, \mathrm{n}=20$ & $\mathrm{k}=18, \mathrm{n}=20$ & $\mathrm{k}=20, \mathrm{n}=30$ \\
\hline \multirow{3}{*}{ Existing model[3] } & LR & 0.0142 & 0.00660 & 0.00873 & 0 & 0.00508 \\
\hline & $\mathrm{RR}$ & 0.787 & 0.786 & 0.820 & 0.546 & 0.862 \\
\hline & MP & 2.58 & 3.05 & 7.78 & 9.28 & 16.71 \\
\hline \multirow{3}{*}{ Prop. model 1} & LR & 0.0547 & 0.0426 & 0.0695 & 0.0111 & 0.0528 \\
\hline & $\mathrm{RR}$ & 0.480 & 0.623 & 0.486 & 0.380 & 0.468 \\
\hline & MP & 1.45 & 2.26 & 4.37 & 6.46 & 8.89 \\
\hline \multirow{3}{*}{ Prop. model 2} & LR & 0.0935 & 0.0720 & 0.144 & 0.0730 & 0.160 \\
\hline & $\mathrm{RR}$ & 1.00 & 1.00 & 0.997 & 1.00 & 1.00 \\
\hline & MP & 3.9 & 6.99 & 13.9 & 18.8 & 25.9 \\
\hline \multirow{3}{*}{ Prop. model 3} & LR & 0.104 & 0.275 & 0.272 & 0.0607 & 0.235 \\
\hline & $\mathrm{RR}$ & 0.930 & 1.00 & 1.00 & 0.851 & 0.994 \\
\hline & MP & 3.16 & 11.4 & 13.3 & 14.5 & 21.9 \\
\hline \multirow{3}{*}{ Prop. model 4} & LR & 0.0942 & 0.254 & 0.251 & 0.0819 & 0.210 \\
\hline & $\mathrm{RR}$ & 1.00 & 1.00 & 1.00 & 1.00 & 1.00 \\
\hline & MP & 3.97 & 14.6 & 17.3 & 19.0 & 28.0 \\
\hline \multirow{3}{*}{ Prop. model 5} & LR & 0.0824 & 0.241 & 0.242 & 0.0307 & 0.179 \\
\hline & $\mathrm{RR}$ & 0.790 & 1.00 & 0.960 & 0.674 & 0.886 \\
\hline & MP & 2.49 & 8.24 & 10.2 & 11.5 & 17.2 \\
\hline \multirow{3}{*}{ Prop. model 6} & LR & 0.101 & 0.229 & 0.233 & 0.0848 & 0.208 \\
\hline & $\mathrm{RR}$ & 1.00 & 1.00 & 1.00 & 1.00 & 1.00 \\
\hline & MP & 3.96 & 13.1 & 16.8 & 19.0 & 27.6 \\
\hline \multirow{3}{*}{ Prop. model 7} & LR & 0.0371 & 0.0655 & 0.0559 & 0.00697 & 0.0381 \\
\hline & $\mathrm{RR}$ & 0.327 & 0.383 & 0.590 & 0.274 & 0.333 \\
\hline & MP & 0.98 & 2.07 & 3.45 & 4.66 & 6.33 \\
\hline \multirow{3}{*}{ Prop. model 8} & LR & 0.0806 & 0.112 & 0.182 & 0.0771 & 0.177 \\
\hline & $\mathrm{RR}$ & 1.00 & 1.00 & 0.999 & 1.00 & 1.00 \\
\hline & MP & 3.92 & 9.12 & 15.0 & 18.9 & 26.5 \\
\hline \multirow{3}{*}{ Prop. model 9} & LR & 0.0851 & 0.106 & 0.221 & 0.0397 & 0.196 \\
\hline & $\mathrm{RR}$ & 0.830 & 0.927 & 0.944 & 0.729 & 0.904 \\
\hline & MP & 2.65 & 4.20 & 9.69 & 12.4 & 17.6 \\
\hline \multirow{3}{*}{ Prop. model 10} & LR & 0.0843 & 0.0867 & 0.192 & 0.0800 & 0.186 \\
\hline & $\mathrm{RR}$ & 1.00 & 0.997 & 1.00 & 1.00 & 1.00 \\
\hline & MP & 3.91 & 6.65 & 14.9 & 18.9 & 26.6 \\
\hline
\end{tabular}

\section{References}

[1] Kawagoe Flower Auction Inc.

http://www.kawagoekaki.co.jp/auction.html (2018/09/20 access)

[2] Konosu flower cener (in Japanese)

http://www.kounosukaki.co.jp/market/bidding.htm (2018/12/24 access)

[3] Minoru Kitahara,Ryo Ogawa(2010),"Efficiency versus Economy of Time in Multi-Unit Descending Auction:The Role of "Mari" at Flower Markets in Japan"

http://www.iser.osaka-u.ac.jp/library/dp/2010/DP0774.pdf 